\title{
Biometrics to Improve Methodologies on Understanding Player's Gameplay Experience
}

\author{
Pejman Mirza-babaei \\ University of Sussex \\ Brighton, BN1 9RH, UK \\ pm75@sussex.ac.uk
}

\begin{abstract}
Due to the specific characteristics of video games most of the established $\mathrm{HCl}$ (Human-Computer Interaction) methods of user research cannot be used the same way for video games. To address this, the research project presented here adapts methods suited for empirical research on video games. This will be based on utilising player's physiological measures in conjunction with other user research methodologies such as observation, interview, think-aloud, heuristics and behavioural coding.
\end{abstract}

Video games, User Experience, Usability, Methodology, Biometrics, mixed-method

\section{INTRODUCTION}

In recent years, there have been many changes in video games development, including new business models, widening player demographics and new controller interfaces. These present opportunities but also additional uncertainties and, in combination with escalating design and development cost, developers are focused on ensuring every game is as big a success as possible. Therefore the demand for studies dealing with the users and their interaction with video games has grown in the last years.

Although $\mathrm{HCl}$ methods have made progress in understanding the usability of productivity applications, applying the current methods to identify user experience (UX) issues in video games is still a challenge for researchers.

Due to the specific characteristics (such as emotion and intentional challenge) of video games most of the well established $\mathrm{HCl}$ methods of user research cannot be used the same way for video games.

The focus of the research project presented here is to introduce new methodologies (mixed-methods) by combining biometric measures with other $\mathrm{HCl}$ methods to identify and communicate user experience issues for gameplay environments. This would enable video game user researchers to have a better understanding of player's gameplay experiences. The hypothesis of this research is that user experience methodologies are of benefit to video game development, with the following questions to be answered: (1) Understanding the limitations of current approaches of using biometrics in video game user research, and how novel research can address these issues. (2)
Applying physiological measures to introduce and develop a practical method that developers can easily understand, design and evaluate player experiences. (3) Exploring the extent to which physiological measures can be adapted for video game user research.

\section{BIOMETRICS IN HCI}

Physiological metrics have recently been used in Human-Computer Interaction. Some researchers have used Galvanic Skin Response (GSR) and cardiovascular measures to examine user response to well and ill-designed web pages (Ward et al., 2003.) Hazlett (2007) have used facial EMG (electromyography) as feedback in the software design process. Other researchers have tried to use GSR as an index of cognitive load. A study by $\mathrm{Yu}$ Shi et al. (2007) showed potential results in explaining the peaks in the GSR data, which they found to correlate with user cognitive load in subtask user events.

Biometrics have also been explored as a measure for evaluating video games usability. Hazlett (2008) describes the use of facial EMG as a measure of positive and negative emotional valence during interactive experience. Ravaja (2006) measured facial EMG and cardiac interbeat intervals in addition to self-report ratings to index physiological arousal and emotional valence.

Some researchers in UX have used physiological measurements to evaluate emotional experience in play environments. Mandryk and Atkins (2007) described an experiment designed to test the efficacy of physiological measures as evaluators of 
collaborative entertainment technologies, where they have examined physiological responses to different interactive play environment. Nacke (2009) created a real-time emotional profile (flow and immersion) of gameplay. His results demonstrate a correlation between subjective and objective indicators of gameplay experience and show the potential for providing real-time emotional profiles of gameplay that may be correlated with self-reported subjective descriptions.

Using a response profile for a set of physiological variables enables scientists to go into more detail with their analysis and allows for a better correlation between response profile and psychological event (Cacioppo et al., 2007.) However, changes in the physiological signals can be responses to external activity or can be in anticipation of something not otherwise observed. Moreover, specific types of measurement of different responses are not trustworthy signs of feeling. The often-described 'many-to-one' relation between psychological processing and physiological response allows for physiological measures to be linked to a number of psychological structures (Cacioppo et al., 2007.) Ambinder states; "Some responses or measurements are difficult to correlate with something specific that happened in the game" (Onyett, 2009.)

\section{BIOMETRIC AND POST-SESSION INTERVIEW}

In our first approach we proposed a procedure that enables video games user researcher to identify events in a game with higher impact on the players' feeling (Mirza-babaei \& McAllister, 2010.) In this approach we capture player's physiological measures (e.g. GSR) during gameplay sessions, to indicate micro-events when players were aroused. We are not interested to over-interpret physiological measures into particular emotion, but, rather to use them to structure the post-gameplay interview to ask players to comment and describe their feelings during the selected events. This approach would be able to identify not only the negative usability and user experience issues but also the events in the game which have a positive impact on player experience. These events may go unnoticed if using traditional methods only. The post-gameplay interview was conducted immediately after they had finished the gameplay session so that the participants could remember most of their actions and thoughts. With the participants, we looked at selected moments of their gameplay video and they described their feelings about those moments but most importantly why they felt that way.

Our study aims to quantify the value of this approach as an addition to traditional observationbased user research methodologies. Our result showed that observation-based techniques can expose the majority of issues relating to usability, however this approach enabled researchers to discover latent issues in related to players' feelings, immersion and gameplay experience and, in certain categories of issue, can reveal over $50 \%$ more issues than observation alone (Mirza-babaei et al., 2011.)

\section{BIOMETRIC STORYBOARDS}

Our next approach is on introducing a new technique called 'Biometric Storyboards' (Mirzababaei \& McAllister, 2011.) Narratives have always been part of the user experience process to communicate how and why a design would work (Quesenbery et al., 2010). We are promoting an approach based on using stories (or storyboards,) where we graph the player's gameplay experience over a longer period (e.g. a level of a game.)

The graph itself is drawn based on (1) player's biometric responses (2) player post-session interviews to explain 'why' the change in their signal occurred (3) players' self-drawn diagrams of their gameplay experience and (4) coding player gameplay behaviour (or context.)

Sometimes the limitations of stories are that they are a personal and subjective experience, from the perspective of the consumer. Thus, they become a fairly intangible experience to record. Using our proposed approach of the biometric storyboard, the seemingly elusive narrative experience becomes a data-supported recorded asset.

\subsection{Players' self-drawn diagrams}

Player drawn diagrams enable us to capture a player's overall experience of each level. We saw that players can mostly recall details from the beginning and end of their gameplay session -serial position effect (Healy, 2000.) (Figure 1)

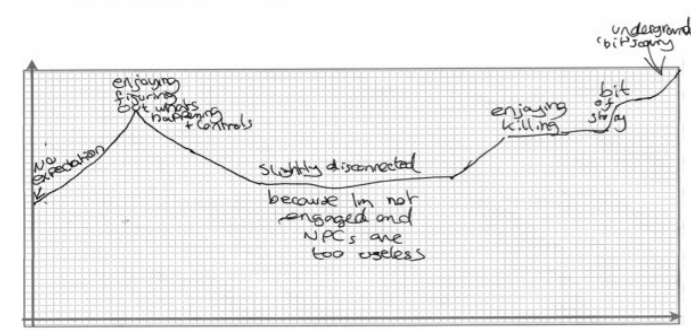

Figure 1: Example of player drawn diagrams of their gameplay experience

Combining the player's drawing and biometrics with structured post-session interview helps us to address this problem.

\subsection{Player gameplay behaviour}

To help us better represent an accurate reflection of the player's gameplay experience, we include 
their gameplay behaviour when generating the biometric storyboard. This helps us to revel the connection between game design and player gameplay behaviour and feeling.

Observational based approaches focus on what players do, but do not address the player experience issues of 'why they did it', or 'how they felt'. This technique focuses on understanding players and gameplay by analysing the coupling between behaviour (what they did) and its resonating response in the biometrics reading (how they felt).

\subsection{First experiment}

An unreleased commercial single player first person shooter console game was subjected to single player user testing for approximately one hour per player, and with 9 players: 5 self-identified mainstream gamers and 5 core gamers. Only a portion of the game was complete to a level of quality indicative of the final product, and only these sections were tested. Testing was conducted over 3 days in laboratory conditions. Participants played the game on an Xbox 360 connected to an HD television. Video cameras recorded the player, biometrics kit capturing GSR, and realtime footage from the game console was simultaneously streamed to the observation room next door. All feeds were composited together on a single display, and recorded for later analysis. The game's producer and experiment conductors monitored the participants' play from the observation room. The experiment conductors had spent some time familiarising themselves with the game before the test sessions, and the producer was able to identify when players were not playing the game as intended. Following the user test sessions, a biometric storyboard was produced of each level for a casual and core player.

Figure 2 shows an example diagram. Each vertical line is one minute of gameplay, positive comments are in green and negative are in red. It shows in both levels that minutes 10-20 offer a poor experience, and gamers experience frustration and lack of enjoyment.

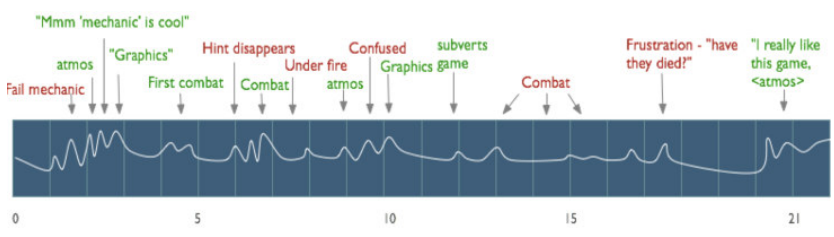

Figure 2: Example of Biometric storyboards (old design).

\subsection{Second experiment}

The second experiment was conducted under similar conditions to the first, but with a different set of 9 participants. The new version of biometric storyboards was refined based on the feedback from the game's producer. The feedback suggested that the first design was difficult to compare between players.

Figure 3 (below) shows the new design for the second experiment. The following are the main differences as: 1) each level was divided into thematic areas, this would make the key sections easier to compare; it also shows the time it took the player to complete that area. Also, it makes it easier for game designers to see where the issues are exactly. 2) Brief text explanation on player's experience story (with reference to arousal events). 3) Green or red dots shows the positive or negative experience (mainly from the player's drawing). 4) Screenshot of game events (removed from the below diagram since the game is unreleased).

Once we've plotted a series of these, we can compare the gameplay journey of users and employ them to spot key trends. Our primary result shows that the players' background profiles (e.g. hardcore or casual player) and 'psychographics' (motivation) reflect a regular pattern in their corresponding biometric storyboards.

\section{Biometric Storyboard Player 3 (core) Level 1}

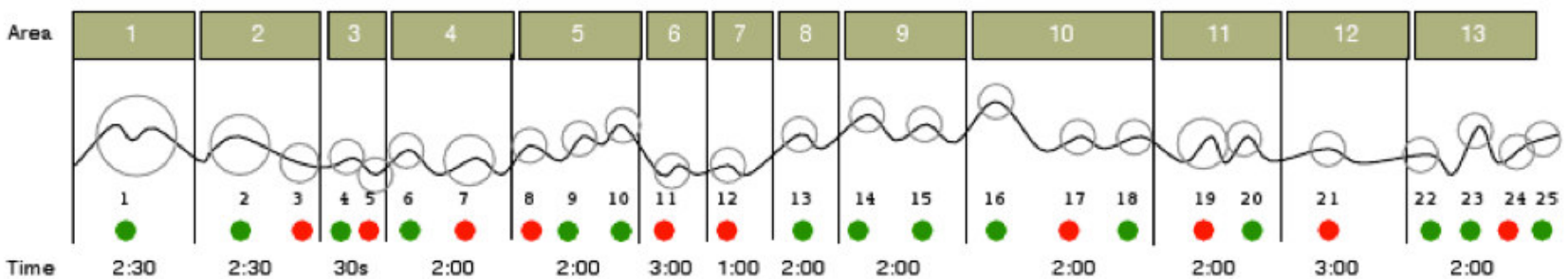

Figure 3: Biometric storyboards (new design).

\section{CONCLUSIONS AND FURTHER WORK}

Early results from pilot studies suggest that these approaches improve our understanding of the players' gameplay experiences, but they have their own limitations and need to be developed.

To increase the accuracy of the 'biometric storyboard' approach, we want to include player's gameplay behaviour coding. This should also help 
us to be able to explain 'why' the player experienced certain emotions.

We have seen a different pattern in each player's biometric storyboard reflects on the player's profile but this is yet to be studied and validated.

Of further interest is the differing genres of video games, and the associated types of issue and their biometric expression in participants. For the results of this and further studies to be generalisable to all video game genres, it is important that all game types be represented. Further research may also indicate that certain biometric sensors are better suited to the extraction of usability and player experience issues for certain genres.

The mixed-method approaches generated as a result of this research has been evaluated and iterated on unreleased commercial video games. We had positive feedback from game producers on how these approaches helped them to have a better understanding of players' interaction with their game. This ultimately enhances their ability to effectively optimise the experience of the final release.

\section{ACKNOWLEDGMENT}

Thank to Geraldine Fitzpatrick, Graham McAllister and Gareth White for great support and valuable discussions. I would also like to thank Emma Foley and Sebastian Long for research assistance.

\section{REFERENCES}

Cacioppo, J. T., Tassinary, L. G., \& Berntson, G. G. (2007) Psychophysiological Science. Handbook of psychophysiology.

Calvert, R. M. (2006) Using psychophysiological techniques to measure user experience with entertainment technologies. Behaviour \& Information Technology.

Hazlett, R. L. (2007) Measuring emotional valence to understand the user's experience of software. Internationa Journal of Human-Computer Studies, 65, 306-314.

Hazlett, R. (2008) Using Biometric Measurement to Help Develop Emotionally Compelling Games. In K. a. Isbister, Game Usability: Advancing the Player Experience. Morgan Kaufmann, MA, USA.

Healy, A. H. (2000) Comparing serial position effects in semantic and episodic memory using reconstruction of order tasks. Journal of Memory and Language, 42, 147-167.

Mandryk, R. L., \& Atkins, M. S. (2007). A fuzzy physiological approach for continuously modeling emotion during interaction with play technologies. International Journal of Human-Computer Studies, 329-347.

Mirza-babaei, P. \& McAllister, G., (2010) Using Biometrics to better understand the players' gameplay experience. Conference presentation at Future Game On. Paris, France, 9-10 Sep 2010.

Mirza-babaei, P. \& McAllister, G., (2011) Biometric Storyboards: visualising meaningful gameplay events. Brain and Body Interface CHI 2011 Workshop. Vancouver, Canada, 8 May 2011.

Mirza-babaei, P., Long, S., Foley, E. \& McAllister, G., (2011) Understanding the Contribution of Biometrics to Games User Research. Full paper accepted for publication at DiGRA 2011, Utrecht, The Netherlands, 14-17 September 2011.

Nacke, L. (2009). Affective Ludology. Scientific Measurement of User Experience in Interactive Entertainment. Game Systems and Interaction Research Laboratory, School of Computing Blekinge Institute of Technology.

Onyett, C. (2009) GDC 09: Why Are Valve's Games So Polished?. http://uk.pc.ign.com/articles/966/966972p1.html (Retrieved January 5, 2011) Quesenbery, W., \& Brooks K., (2010) Storytelling for User Experience. Louis Rosenfeld, New York. Ravaja, N. (2006) Spatial Presence and Emotions during Video Game Playing: Does It Matter with Whom You Play?. Virtual Environment, 15, 381392.

Shi, Y. R. et al. (2007) Galvanic Skin Response (GSR) as an Index of Cognitive Load. CHI 2007, 2651-2656. ACM.

Ward, R. D., \& Marsden, P. H. (2003) Physiological responses to different web page design. International Journal of Human-Computer Studies, 199-212. 